(C) 2014 IEEE. Personal use of this material is permitted. Permission from IEEE must be obtained for all other uses, in any current or future media, including reprinting/republishing this material for advertising or promotional purposes, creating new collective works, for resale or redistribution to servers or lists, or reuse of any copyrighted component of this work in other works.

This paper appears in: Communications and Information Technologies (ISCIT), 2014 14th International Symposium on, Issue Date: 24-26 Sept. 2014.

The definitive version is available online at: http://dx.doi.org/10.1109/ISCIT.2014.7011956. 


\section{LTE-Advanced, and the Way Forward}

\author{
Mohammad Dehghani \\ Electrical, Electronic and Computer Engineering \\ University of Greenwich, \\ Chatham, Kent, United Kingdom \\ Email: arash.dehghani@gmail.com
}

\author{
Kamran Arshad \\ Electrical, Electronic and Computer Engineering \\ University of Greenwich, \\ Chatham, Kent, United Kingdom \\ Email:k.arshad@gre.ac.uk
}

\begin{abstract}
Long Term Evolution Advanced (LTE-Advanced) is the next step in LTE evolution and allows operators to improve network performance and service capabilities through smooth deployment of new techniques and technologies. LTE-Advanced uses some new features on top of the existing LTE standards and protocols to provide better user experience and higher throughputs. Some of the most significant features introduced in LTE-Advanced are carrier aggregation, enhancements in heterogeneous networks, coordinated multi-point transmission and reception, enhanced Multiple Input Multiple Output (MIMO) usage and deployment of relay nodes in the radio network. This paper presents an overview of the key features and functionalities of the LTE-Advanced radio access network supported by simulation results, and provides discussion on the current challenges, roadmap and the way forward from LTEAdvanced toward future mobile communications systems.
\end{abstract}

Keywords - LTE-Advanced; Carrier Aggregation; MIMO; Heterogeneous Network; Relay Nodes; CoMP Transmission and Reception;

\section{INTRODUCTION}

Wireless mobile data traffic has increased tremendously in the last few years and is expected to increase 13-fold between 2012 and 2017 [1]. The leading driver for this increased growth of wireless data traffic, especially in cellular communications, is Smartphones [1]. This increasing demand is pushing the existing wireless mobile networks to their limits, causing a reduction in data throughput, decreasing the availability of resources and increasing data transmission delay.

Long Term Evolution of 3G (LTE) is one of the strongest candidates proposed by the $3^{\text {rd }}$ Generation Partnership Project (3GPP) for deploying globally as the next generation of wireless mobile networks (i.e. 4G). LTE has been the fastest developing mobile system to date and as of July 2014, 318 operators have commercially launched LTE systems worldwide [2]. In LTE release 10 (also referred as LTEAdvanced), significant changes have been made in the radio capabilities and the peak and achievable data rates improved significantly.

LTE-Advanced is meant to fulfil the requirements of International Telecommunication Union - Radio communication sector (ITU-R) for International Mobile Telecommunication - Advanced (IMT-Advanced). Main features and techniques deployed in LTE-Advanced physical

The research leading to these results was derived from the University of Greenwich Research \& Enterprise Investment Programme grant under agreement number RAE-ES-01/14. layer (PHY) can be summarised as follows:

- More bandwidth using Carrier Aggregation (CA);

- Using more antennas and new antenna techniques in the uplink and downlink transmission (MIMO enhancement);

- Cooperation between cells and Coordinated Multi Point (CoMP) transmission and reception;

- Using small cells and relays;

In section II of this paper the specifications of LTE and LTE-Advanced are presented. Section III describes the mentioned enhancements and features. Simulation results for LTE-Advanced enhancements and features are presented in section IV following with the discussions regarding the challenges and future roadmap of LTE-Advanced in section V. And finally, the paper is concluded in section VI.

\section{LTE SYSTEMS OVERVIEW}

LTE release 8 and 9 meet the IMT-Advanced requirements in several areas such as high mobility support and latency. However, 3GPP set tight requirements compared to IMTAdvanced 'for LTE-Advanced in order to confidently meet all the IMT-Advanced requirements and make considerable improvements from release $8 / 9$ to release 10 [3]. Some of the main physical layer features in release 10 and subsequent releases of LTE-Advanced (i.e. release 11 and 12) are summarised in Table I.

TABLE I. LTE-ADVANCED FEATURES

\begin{tabular}{|c|c|c|}
\hline Release 10 & Release 11 & Release 12 \\
\hline Carrier aggregation & $\begin{array}{c}\text { Carrier aggregation } \\
\text { enhancement }\end{array}$ & $\begin{array}{c}\text { MIMO } \\
\text { enhancement }\end{array}$ \\
\hline Advanced MIMO & $\begin{array}{c}\text { CoMP } \\
\text { CoMP } \\
\text { transmission/reception }\end{array}$ & \multirow{2}{*}{$\begin{array}{c}\text { Small cells } \\
\text { enhancement }\end{array}$} \\
\hline $\begin{array}{c}\text { Heterogeneous } \\
\text { network and eICIC }\end{array}$ & Further eICIC (FeICIC) \\
\cline { 1 - 2 } Relay nodes & $\begin{array}{c}\text { Enhanced downlink } \\
\text { control channel }\end{array}$ & \\
\hline
\end{tabular}

\section{ENHANCEMENS AND FEATURES}

\section{A. Carrier Aggregation}

$\mathrm{CA}$ is considered to be the most important and effective feature of LTE-Advanced. The key idea in CA is to provide wider transmission bandwidth for users by aggregating two or 
more release 8 LTE carriers. In LTE-Advanced, backward compatibility is achieved by aggregating several LTE carriers to increase transmission bandwidth without significant changes to the LTE carriers. With CA, it is also possible to aggregate different carriers from different bands. Carrier aggregation is exciting for mobile network operators as large continuous bandwidths (i.e. on the order of $100 \mathrm{MHz}$ ) are scarce in several countries including the United Kingdom; The network operators may aggregate several small bandwidths distributed among different frequency bands and provide larger bandwidths and the required QoS.

From the theoretical point of view, release 10 signalling supports aggregation of up to five carriers and have a bandwidth of $100 \mathrm{MHz}$ (i.e. $5 \times 20 \mathrm{MHz}$ ) [4]. Due to the practical limitation of the devices, currently only the aggregation of two carriers is considered in release 10 .

\section{B. MIMO Enhancement}

MIMO is a key technology in wireless communications that has potential to increase the capacity by using multiple transmitter and receiver antennas. This feature is currently used in IEEE $802.11 \mathrm{n}, 802.16 \mathrm{~d} / \mathrm{e}$ systems and supported by LTE release 8. MIMO has already been deployed widely in LTE networks, and further enhancements were proposed in release 10. In LTE-Advanced, Single User MIMO (SU-MIMO) is extended to support eight transmit antennas while the maximum number of transmit antennas in release 8 is four. In [5], performance evaluation of LTE-Advanced systems using 8 by $8 \mathrm{MIMO}$ and $20 \mathrm{MHz}$ of bandwidth shows that in an indoor scenario the median throughput up to $335 \mathrm{Mbps}$ can be achieved. The eight transmit antennas at the base station can also be deployed in the transmission diversity scenarios. Furthermore, LTE-Advanced brings enhancements of Multi User MIMO (MU-MIMO) and increases the network capacity that is not achievable in SU-MIMO systems.

\section{Heterogeneous Network (HetNet)}

The HetNet refers to a network that contains macro base stations together with small base stations such as Micro, Pico and Femto cells, simultaneously. Such small base stations are typically deployed within the umbrella of the macro cell coverage.

One of the main challenges in HetNet is to find suitable spectrum for small cells; since all the available spectrum is usually assigned to the macro cells, different cell layers often have to use the same frequency. This may cause interference issues, which should be managed effectively in HetNet's. $3 \mathrm{GPP}$ release 10 has come up with solutions to solve this issue through enhanced Inter Cell Interference Coordination (eICIC). In this feature, macro cells and small cells are coordinated in the time domain and inter cell interference is avoided by preventing simultaneous transmission.

Another issue in HetNet is that the small cells normally do not carry the desirable amount of traffic as they typically have lower output power. The footprint of a small cell can be increased in LTE-Advanced under a feature called Range Extensions (RE). When RE is in place, positive offset is applied to the small cell measurements and UE will choose the small cell in worse radio conditions than without RE. However, it can result in a relatively bad performance in the UEs camped to the small cells, unless it is used together with interference mitigation features like eICIC. Without eICIC feature, these UEs receive strong interfering signals from the close-by macro cells. Hence, eICIC is one of the key feature for enabling RE deployment in LTE-Advanced.

\section{Relaying}

Relay Node (RN) is a small site that operates between base station and UE. The main characteristic of the RN is that it does not need a separate backhaul, and it is a self-backhauling base station. Relay nodes can be used in a situation when providing backhaul is impractical or expensive. Installing RNs on mobile vehicles such as buses or in High Speed Railways (HSR) is another interesting application of the relays, which is under consideration by 3 GPP and ITU.

From the UE point of view, RN seems as a normal base station. From the base station viewpoint, relay acts as an UE with some special features, and the core network considers RN as an additional sector for the base station. The intention of relaying is to increase data rates by reducing transmitter-toreceiver distance. More advanced deployment of the relays such as cognitive relays, self-organising relays, coordinated/cooperative relay systems, and opportunistic relaying are expected to be used in the future mobile communications networks.

\section{E. CoMP Transmission and Reception}

This framework offers different techniques to have a tight coordination between multiple locally distant radio access network nodes such as base stations and RNs. The coordination can be among the cells served by the same base station (intra base station), or the cells served by different base stations (inter base station). For obvious reasons, the synchronisation among base stations is a necessity for CoMP. CoMP was supposed to be considered as a part of the Release 10 at the beginning, but due to the complexity and unclear realistic gain it was excluded later and moved to release 11 and beyond.

The idea of CoMP is that data will be sent (DL) or received (UL) not only by one cell, but also by multiple cells and base stations. Theoretically, two different versions of CoMP exist [6]. The simple version is Coordinated Scheduling/Beamforming (CS/CB) and the advanced version is Joint Processing (JP). The latter is also known as Cooperative MIMO (C-MIMO). In CS/CB CoMP, transmission is merely done from one cell but the interference is minimised by coordination and proper scheduling among the surrounding cells. In JP CoMP, multiple cells are involved in the transmission and they are coordinated for active interference cancellation. Thus, the interference becomes a useful signal and theoretical channel capacity (Shannon limit) is increased. Generally JP brings more system improvements than $\mathrm{CS} / \mathrm{CB}$ [7].

\section{SiMULATIONS RESULTS}

In this paper, typical LTE and LTE-Advanced scenarios with one base station and one UE are defined and downlink 
data throughputs of the user are simulated for different Signal to Noise Ratio (SNR) values. The general simulation parameters for all the LTE and LTE-Advanced cases are set according to the 3GPP specifications, and key parameters are summarised in Table II.

TABLE II. GENERAL SimUlation PARAMETERS

\begin{tabular}{|c|c|}
\hline Parameter & Value \\
\hline Carrier Frequency & $2.5 \mathrm{GHz}$ \\
\hline Max No. of HARQ Retransmissions & 3 \\
\hline Cyclic Prefix & Normal \\
\hline Channel Type & Flat Rayleigh \\
\hline UE Channel Estimation Method & Perfect \\
\hline Number of Simulated Subframes & 800 \\
\hline
\end{tabular}

Firstly, four moderate cases are simulated for both LTE and LTE-Advanced. UE is assumed to have two receiving antennas, which is currently the case in almost all the practical situations. The results, shown in Fig. 1 and Fig. 2, are for two different bandwidths (i.e. $10 \mathrm{MHz}$ and $20 \mathrm{MHz}$ ) and MIMO configurations (i.e. $2 \times 2$ and $4 \times 2$ ). Table III summarises the key simulation parameters for these cases.

TABLE III. SIMULATION PARAMETERS USED FOR FIG. $1 \& 2$

\begin{tabular}{|c|c|}
\hline Parameter & Value \\
\hline CQI & 7 \\
\hline Modulation & $16 \mathrm{AAM}$ \\
\hline \multirow{2}{*}{ Bandwidth } & $10 \mathrm{MHz}$ \\
& $20 \mathrm{MHz}$ \\
\hline \multirow{2}{*}{ MIMO } & $4 \times 2$, Open Loop Spatial Multiplexing (OLSM) \\
\hline
\end{tabular}

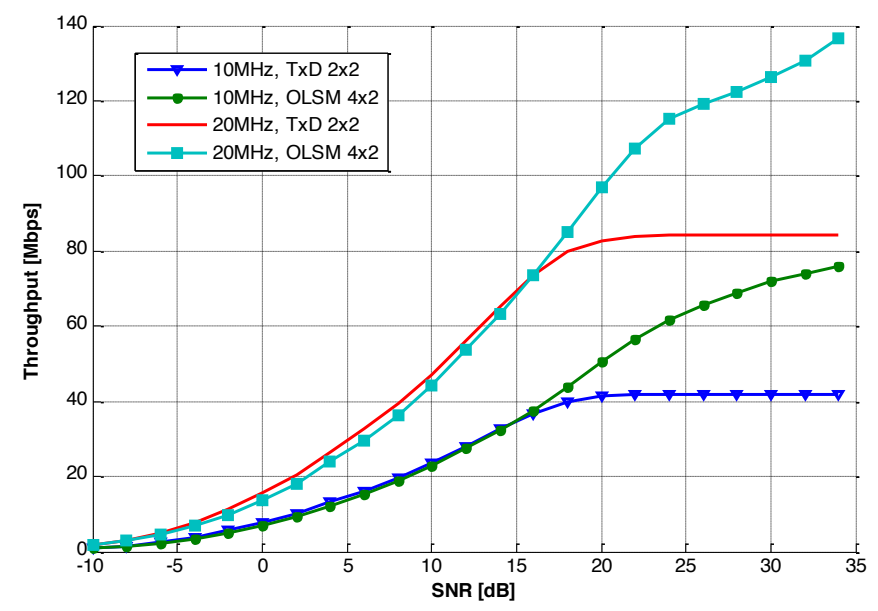

Fig. 2. LTE-Advanced Simulation Results

Secondly, to better illustrate the LTE-Advanced capabilities, a few more cases are defined (summarised in Table IV) and simulated using system level simulations. In these cases, the highest Channel Quality Indicator (CQI) value (i.e. 15) is considered and assumed that the base station has eight transmission antennas and the MIMO operation is in the Closed Loop Spatial Multiplexing (CLSM) mode. Simulation results are presented in Fig. 3.

TABLE IV. Simulation PARAMETERS USED FOR Fig. 3

\begin{tabular}{|c|c|}
\hline Parameter & Value \\
\hline CQI & 15 \\
\hline Modulation & $64 \mathrm{QAM}$ \\
\hline Bandwidth & $20 \mathrm{MHz}$ \\
\hline & $8 \times 2$ \\
MIMO & $8 \times 4$ \\
& $8 \times 8$ \\
\hline
\end{tabular}

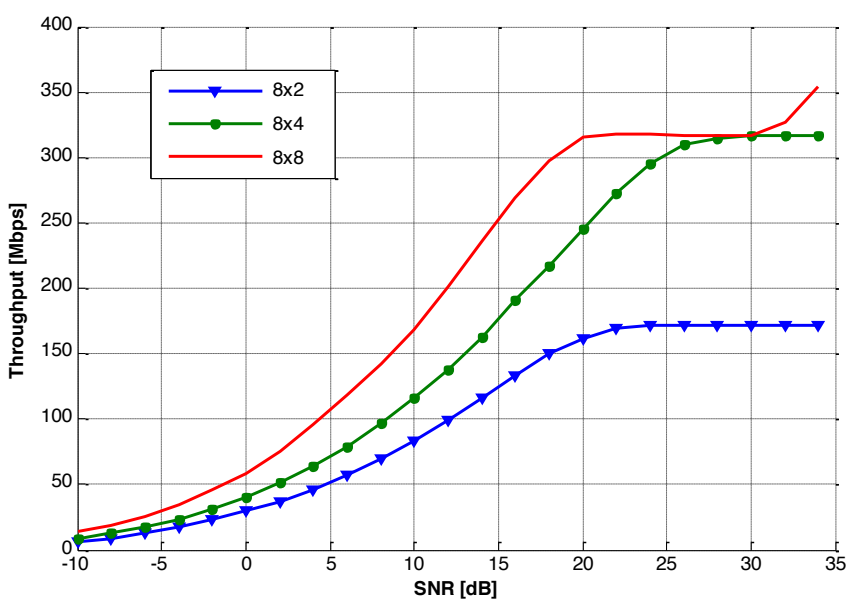

Fig. 3. LTE-Advanced Simulation Results

The throughput can be achieved in LTE-Advanced, as illustrated in Fig. 3, is significant. The improvement in throughput from LTE to LTE-Advanced is quite visible from Fig. 1 and Fig. 2 and LTE-Advanced can be considered is a bold step towards meeting the fast-growing users' demands. It is seen that the advantages of LTE-Advanced over LTE are more substantial for higher SNR and any features that enhance the link quality can help to get more out of LTE-Advanced technology. The features discussed in this article add more enhancements in different aspects such as peak, average and cell edge data throughput and spectral efficiency.

\section{DISCUSSIONS AND ROADMAP}

The actual data rate experienced by users is becoming at the centre of attention instead of peak data rates. Despite all improvements in LTE-Advanced system, cell-edge user data rates are far less than the peak data rates and the difference between cell-edge and peak data rates is too high. Currently, the actual data throughputs dramatically depend on the link quality, and are limited by inter-cell interference. Although frequency reuse-1 systems like LTE and LTE-Advanced are highly efficient in terms of overall efficiency, they suffer from high inter-cell interference at the cell boundaries. Hence, more 
developments on the interference management features such as CoMP transmission and reception, and eICIC are necessary. Furthermore, more enhancements in small cell deployments are needed as they can improve the radio link quality of the users.

The first commercial LTE-Advanced network launched in October 2012 although no LTE-Advanced UE was available at the time. After releasing the first LTE-Advanced chipset in June 2013, several mobile network operators such as SK Telecom and LG Uplus have started to offer LTE-Advanced services to their customers. However, according to the current trends, LTE-Advanced systems are less likely to appear as the mainstream in the markets before 2016.

While 3GPP focuses on the release 12 finalisation, the release 13 study items are getting together and as of now a few items such as Radio Access Network (RAN) sharing enhancements have been set. On the other hand, studies on the beyond-LTE-Advanced systems for the future wireless mobile communications networks - known as $5 \mathrm{G}$ have already started. Considering the previous trends and the time frame between the generations, $5 \mathrm{G}$ is predicted to be in the picture by 2020 and LTE-advanced technology would have to compete with that as well.

ITU-R estimations show that at least $1280 \mathrm{MHz}$ of spectrum bandwidth are required for the future development of IMT-2000 and IMT-Advanced by the year 2020 [8]. The required bandwidth can be provided by allocating new spectrum in higher frequency bands for cellular systems. This may bring new challenges to the networks due to the special characteristic of the shorter wavelength signals. Another solution for dealing with the spectrum scarcity issues is to use Cognitive Radio (CR) technology in the networks in order to enable terminals to opportunistically access the spectrum that is being underutilised. CR technology offers flexible and efficient usage of the existing spectrum bandwidth and CR deployment seems inevitable for further evolution of LTE-Advanced networks. It will also play a more important role in $5 \mathrm{G}$ systems.

\section{CONCLUSION}

Five key features in LTE-Advanced systems are discussed in this paper. Each of them brings some advantages to the wireless mobile networks for both operators and users. CA can provide higher peak data rate and cell edge user experience improvements. MIMO enhancement techniques do the same as well as average data rate enhancement depending on the MIMO configurations and methods. CoMP transmission can improve coverage in noise limited scenarios. HetNet enhances average and cell edge data rates. Regarding the RNs, their achievements depend on the situation they are deployed in; they can provide coverage for some locations that other kinds of base station cannot do, and therefore come up with a huge gain. These features are likely to be enhanced further and considered in future releases and wireless mobile technologies.

\section{ACKNOWLEDGMENT}

The simulations results presented in this paper have been generated using Vienna LTE Link Level Simulator and Vienna LTE Link Level Simulator provided by the Institute of Telecommunications, Vienna University of Technology, Austria.

\section{REFERENCES}

[1] Cisco, "Cisco Visual Networking Index: Global Mobile Data Traffic Forecast Update, 2012-2017," Cisco white paper, 2013.

[2] GSA, "Evolution to LTE Report," Global mobile Suppliers Association, July 2014.

[3] 3GPP TR 36.913, "Requirements for Further Advancements for Evolved Universal Terrestrial Radio Access (EUTRA) (LTE-Advanced)," 3rd Generation Partnership Project (3GPP), 2012.

[4] 3GPP TR 36.814, "Further advancements for E-UTRA physical layer aspects," 3rd Generation Partnership Project (3GPP), 2010.

[5] K. Werner, H. Asplund, D. Figueiredo, N. Jalden and B. Halvarsson, "LTE-advanced $8 \times 8$ MIMO measurements in an indoor scenario," in International Symposium on Antennas and Propagation (ISAP), Nagoys, 2012.

[6] D. Lee, H. Seo, B. Clerckx, E. Hardouin, D. Mazzarese, S. Nagata and K. Sayana, "Coordinated multipoint transmission and reception in LTEadvanced: deployment scenarios and operational challenges," IEEE Communications Magazine, vol. 50, no. 2, pp. 148 - 155, 2012.

[7] Y.-H. Nam, L. Liu, Y. Wang, C. Zhang, J. Cho and J.-k. Han, "Cooperative communication technologies for LTE-advanced," in IEEE International Conference on Acoustics Speech and Signal Processing (ICASSP) , Dallas, TX, 2010.

[8] ITU-R M.2078, "Estimated spectrum bandwidth requirements for the future development of IMT-2000 and IMT-Advanced," 2006. 